\section{Efecto del nivel socioeconómico sobre la mortalidad en áreas urbanas: revisión crítica y sistemática}

\author{
Effect of socioeconomic status on mortality in \\ urban areas: a systematic critical review
}

\author{
Efeito do status socioeconômico na mortalidade \\ em áreas urbanas: análise crítica sistemática
}

\author{
1 Gerencia de Gestión \\ Integrada de Vigo, Servicio \\ Gallego de Salud, Vigo, \\ España. \\ 2 Departamento de Medicina \\ Preventiva y Salud Pública, \\ Universidad de Santiago de \\ Compostela, Santiago de \\ Compostela, España. \\ Correspondencia \\ A. Salgado-Barreira \\ Unidad de Apoyo a la \\ Investigación, Gerencia de \\ Gestión Integrada de Vigo, \\ Servicio Gallego de Salud. \\ Hospital do Meixoeiro, \\ planta 2. Meixoeiro s/n, Vigo/ \\ Galicia - 36214, España. \\ angel.salgado.barreira@ \\ sergas.es
}

\begin{abstract}
Socioeconomic inequalities cause more disease and death than most risk factors, especially in cities. This systematic review of the scientific evidence included articles in English, Spanish, Portuguese, and Italian and excluded studies with low levels of evidence and those that did not analyze associations between mortality and socioeconomic status in urban settings. Articles were selected by two independent reviewers, and data extraction used evidence tables. A total of 1,509 records were obtained, and 24 were included. All the studies showed higher mortality rates in poorer areas. Six studies showed an association with cardiovascular diseases, four with lung diseases, and three with AIDS, infectious and parasitic diseases, and cirrhosis. The selected studies showed low consistency in the results and important methodological limitations that prevented comparisons between studies or the extraction of relevant conclusions.
\end{abstract}

Urban Health; Mortality; Health Inequalities; Socioeconomic Factors
Ángel Salgado-Barreira 1,2

Ana Estany-Gestal 2

Adolfo Figueiras 2

\section{Resumen}

Las desigualdades socioeconómicas son una causa de mortalidad y morbilidad superior a la mayoría de factores de riesgo, especialmente en el entorno urbano. Se llevó a cabo una revisión sistemática de la evidencia científica, en la que se incluyó artículos en inglés, castellano, portugués e italiano y se excluyeron estudios de baja evidencia, y en los que no se analizaba la relación entre mortalidad y nivel socioeconómico en un entorno urbano. La selección de artículos se llevó a cabo por dos revisores independientes y la extracción de datos se realizó con tablas de evidencia. Se obtuvieron 1.509 registros y se incluyeron 24. En todos los trabajos se observó mayor mortalidad en las áreas con peores indicadores de privación. Se observó asociación con patologías cardiovasculares en seis estudios, en cuatro con patologías pulmonares y en tres con SIDA, infecciones y parasitosis y cirrosis. Los estudios incluidos presentan resultados poco consistentes y limitaciones metodológicas importantes que impiden la comparación entre estudios y la extracción de conclusiones relevantes.

Salud Urbana; Mortalidad; Desigualdades en la Salud; Factores Socioeconómicos 


\section{Introducción}

La comprensión del desarrollo económico, social, político y sanitario de un país pasa por conocer las transformaciones que se producen en las grandes ciudades que lo conforman 1. Estas grandes ciudades han asimilado la pobreza en barrios marginales, siendo estos barrios donde se detectan los peores indicadores socioeconómicos y de salud, como, por ejemplo, altas tasas de SIDA, tuberculosis o sobredosis por drogas 2 . El impacto de las desigualdades sociales y económicas dentro de una misma ciudad es enorme y actualmente se considera uno de los principales factores de riesgo de enfermar.

Por otra parte, en la mayoría de países en los que se ha estudiado este fenómeno, se ha determinado que los indicadores de salud son mejores en las zonas urbanas más favorecidas, hecho que incrementa las desigualdades socioeconómicas 3 , entendidas como las diferencias existentes en la distribución y acceso a recursos sociales y económicos, entre las diferentes áreas de una ciudad o área urbana. Hoy en día se dispone de evidencias que demuestran que las desigualdades en salud son evitables, ya que pueden reducirse mediante políticas públicas sanitarias y sociales ${ }^{4}$.

La descripción de las desigualdades en los distintos territorios de una ciudad tiene importancia, tanto para los investigadores, como para los planificadores y las administraciones sanitarias 5,6. Aunque los estudios de desigualdades en áreas pequeñas suelen relacionar las medidas de salud con las medidas de privación, hasta el momento no existen revisiones sistemáticas en la literatura científica que estudien de manera conjunta toda esta información. Además, todavía no existe información detallada sobre la metodología más adecuada para llevar a cabo este tipo de estudios que valoran la relación entre el nivel socioeconómico y la mortalidad.

El objetivo de este trabajo es valorar el estado actual del estudio de la relación entre las desigualdades socioeconómicas y la mortalidad en zonas urbanas.

\section{Método}

\section{Búsqueda bibliográfica}

Para dar respuesta a los objetivos de esta revisión sistemática de la literatura científica, se llevó a cabo una búsqueda de toda la evidencia existente hasta junio de 2012 en MEDLINE, EMBASE, IME (Índice Médico Español), ICYT (Índice de Ciencias y Tecnologías) e ISOC (Índice de Ciencias Sociales y Humanidades). La estrategia de búsqueda utilizada incluyó los términos "mortality" AND "socioeconomic factors" AND ("cities" OR “urban population”).

Con posterioridad se realizó una búsqueda ascendente a partir de la bibliografía de los artículos incluidos. Este proceso se completó mediante una búsqueda general en Internet (organizaciones, sociedades científicas...), con el fin de encontrar otra información de interés. Por último, los resultados de las búsquedas realizadas en las diferentes bases de datos se volcaron en el gestor de datos EndNote X1 (Thomson Reuters, Estados Unidos), para la detección y posterior eliminación de las referencias duplicadas.

\section{Criterios de inclusión y exclusión}

Se incluyeron artículos escritos en inglés, castellano, portugués o italiano, y fueron excluidos los siguientes tipos de estudios, revisiones narrativas, editoriales, artículos de opinión, cartas al director, estudios de un solo caso y comunicaciones a congresos.

También se excluyeron los estudios que, a pesar de incluirse en los resultados de la búsqueda, su objetivo no era analizar la relación entre la mortalidad y el nivel socioeconómico en entornos urbanos.

\section{Selección de los estudios}

Los estudios fueron seleccionados por dos revisores (A.S.-B. y A.E.-G.), actuando de forma independiente y con el uso de un método estandarizado. Los trabajos fueron seleccionados sobre la base de los criterios anteriormente descritos, y en caso de desacuerdo entre los evaluadores, la inclusión fue evaluada por un tercero (A.F.).

\section{Análisis de la información}

La extracción de los datos se llevó a cabo previa elaboración de una tabla específica. Posteriormente, se crearon tablas (Tablas 1 y 2) en las que se recogió la información más relevante de los artículos incluidos: autor principal, año de publicación, periodo y localización del estudio, unidades/áreas en las que se divide la ciudad, tipo de indicador socioeconómico utilizado, medida de la mortalidad y principales resultados obtenidos.

La mortalidad por áreas se evaluó teniendo en cuenta las principales causas de muerte analizadas en los estudios incluidos. No se pudieron realizar metaanálisis y análisis de sensibilidad por la heterogeneidad, a la hora de recoger los datos de mortalidad y nivel socioeconómico. 
Tabla 1

Resultados de los estudios incluidos.

\begin{tabular}{|c|c|c|c|c|c|c|}
\hline Referencia & Año & Ciudad (País) & $\begin{array}{l}\text { Período de } \\
\text { estudio }\end{array}$ & $\begin{array}{l}\text { Área de } \\
\text { estudio }\end{array}$ & $\begin{array}{l}\text { Medida de nivel } \\
\text { socioeconómico * }\end{array}$ & Resultados \\
\hline Pringle et al. 20 & 1983 & $\begin{array}{l}\text { Belfast } \\
\text { (Irlanda) }\end{array}$ & 1970 & Zona (97) & 1 & $\begin{array}{l}\text { Alta correlación entre razón de mortalidad } \\
\text { estandarizada y bronquitis, moderada con neumonía } \\
\text { y lesiones vasculares. } \\
\text { Mayor incidencia de bronquitis y cáncer de pulmón } \\
\text { en nivel socioeconómico bajo y mayor incidencia } \\
\text { de cardiopatías y lesiones vasculares en nivel } \\
\text { socioeconómico alto. }\end{array}$ \\
\hline Byth et al. 7 & 1992 & $\begin{array}{c}\text { Sídney } \\
\text { (Australia) }\end{array}$ & 1979-1983 & $\begin{array}{l}\text { Área de gobierno } \\
\text { local (SLA) (37) }\end{array}$ & 2 & $\begin{array}{l}\text { Correlación entre razón de mortalidad estandarizada } \\
\text { y clase social. }\end{array}$ \\
\hline $\begin{array}{l}\text { Martin Santos } \\
\text { et al. } 21\end{array}$ & 1992 & $\begin{array}{l}\text { Málaga } \\
\text { (España) }\end{array}$ & 1988 & Barrio (91) & 3 & $\begin{array}{c}\text { Todos los indicadores sanitarios, incluida la razón de } \\
\text { mortalidad estandarizada, mejores en el área con } \\
\text { mayor nivel socioeconómico. }\end{array}$ \\
\hline Siskind et al. 8 & 1992 & $\begin{array}{l}\text { Brisbane } \\
\text { (Australia) }\end{array}$ & $1980-1987$ & Área pequeña & 2 & $\begin{array}{l}\text { Hombres: la mortalidad en las } 3 \text { áreas con mejor } \\
\text { nivel socioeconómico fue } 74,1 / 10^{4}( \pm 8,9) \text { mayor que } \\
\text { en nivel socioeconómico bajo. } \\
\text { Mujeres: la mortalidad en las } 3 \text { áreas con mejor nive } \\
\text { socioeconómico fue } 36,8 / 10^{4}( \pm 6,0) \text { mayor que en } \\
\text { nivel socioeconómico bajo. }\end{array}$ \\
\hline Arias et al. 22 & 1993 & $\begin{array}{l}\text { Barcelona y } \\
\text { Valencia } \\
\text { (España) }\end{array}$ & $1983-1985$ & $\begin{array}{l}\text { Barcelona (36); } \\
\text { Valencia (60) }\end{array}$ & 3 & $\begin{array}{l}\text { Barcelona: la razón de mortalidad estandarizada se } \\
\text { correlacionó con desempleo, analfabetismo e } \\
\text { índice de pobreza. } \\
\text { Valencia: la razón de mortalidad estandarizada se } \\
\text { correlacionó con desempleo, número de coches/105 } \\
\text { habitantes e índice de pobreza. }\end{array}$ \\
\hline Bock \& Gans 9 & 1993 & $\begin{array}{l}\text { Ciudad de Panamá } \\
\text { (Panamá) }\end{array}$ & $\begin{array}{l}1981-1986 \\
\text { excepto } 1982 \\
\text { y } 1985\end{array}$ & $\begin{array}{c}\text { Unidad } \\
\text { administrativa (13) }\end{array}$ & 2 & $\begin{array}{c}\text { Diferencias significativas en la mortalidad por causas } \\
\text { pulmonares y otras cardiopatías }(p<0,040) \text { y para } \\
\text { neumonía }(p<0,034) \text { entre los tres } \\
\text { niveles socioeconómicos. }\end{array}$ \\
\hline Fang et al. 30 & 1995 & $\begin{array}{c}\text { New York } \\
\text { (Estados Unidos) }\end{array}$ & 1988-1992 & Vecindad (58) & 4 & $\begin{array}{l}\text { Mayor mortalidad en South Bronx que en el resto de } \\
\text { New York para todos los grupos de edad y } \\
\text { sexo, excepto en enfermedades del corazón y } \\
\text { neoplasias (en > } 75 \text { años). } \\
\text { El cálculo de los años potenciales de vida perdidos } \\
\text { fue muy superior en el South Bronx. }\end{array}$ \\
\hline Reijneveld 10 & 1995 & $\begin{array}{l}\text { Amsterdam } \\
\text { (Holanda) }\end{array}$ & $1986-1990$ & Municipio (22) & 1 & $\begin{array}{l}\text { Hombres: las zonas de bajos ingresos mayor razón } \\
\text { de mortalidad estandarizada por enfermedades } \\
\text { definidas y causas externas. } \\
\text { Mujeres: las zonas de bajos ingresos mayor razón de } \\
\text { mortalidad estandarizada por causas relacionadas } \\
\text { con el tabaco y enfermedades definidas. }\end{array}$ \\
\hline $\begin{array}{l}\text { Akerman } \\
\text { et al. } 11\end{array}$ & 1996 & $\begin{array}{l}\text { São Paulo } \\
\text { (Brasil) }\end{array}$ & 1992 & Distrito (56) & 2 & $\begin{array}{l}\text { Las zonas con peor nivel socioeconómico presentan } \\
\text { mayor mortalidad por patologías circulatorias, } \\
\text { respiratorias, infecciosas, causas externas y todas } \\
\text { las causas. El riesgo fue mayor a menor nivel } \\
\text { socioeconómico. }\end{array}$ \\
\hline $\begin{array}{l}\text { Brimblecombe } \\
\text { et al. } 23\end{array}$ & 1999 & $\begin{array}{c}\text { Oxford } \\
\text { (Inglaterra) }\end{array}$ & 1986-1992 & Área electoral (15) & 3 & $\begin{array}{c}\text { Correlación de la razón de mortalidad estandarizada } \\
\text { con \% de desempleo y nivel de ingresos, tanto en } \\
\text { mujeres como hombres. } \\
\text { No hubo correlación entre la razón de mortalidad } \\
\text { estandarizada y el índice Townsend. }\end{array}$ \\
\hline
\end{tabular}

(continua) 
Tabla 1 (continuación)

\begin{tabular}{|c|c|c|c|c|c|c|}
\hline Referencia & Año & Ciudad (País) & $\begin{array}{c}\text { Período de } \\
\text { estudio }\end{array}$ & $\begin{array}{l}\text { Área de } \\
\text { estudio }\end{array}$ & $\begin{array}{l}\text { Medida de nivel } \\
\text { socioeconómico * }\end{array}$ & Resultados \\
\hline $\begin{array}{l}\text { Middelkoop et } \\
\text { al. } 12\end{array}$ & 2001 & $\begin{array}{l}\text { The Hague } \\
\text { (Holanda) }\end{array}$ & 1982-1991 & $\begin{array}{c}\text { Distrito (93); } \\
\text { Área (27) }\end{array}$ & 2 & $\begin{array}{l}\text { Exceso de mortalidad en las zonas más deprimidas. } \\
\text { Hombres: cardiopatía isquémica }(17,3 \%), \text { patologías } \\
\text { circulatorias (10,2\%), lesión y envenenamiento }(8,6 \%) \text {, } \\
\text { enfermedades hepáticas y cirrosis }(7,2 \%) \text {, } \\
\text { homicidio (6,4\%). } \\
\text { Mujeres: cardiopatía isquémica ( } 25,5 \%) \text {, otras } \\
\text { enfermedades (20,1\%), enfermedades circulatorias } \\
(11 \%) \text {, diabetes }(9,1 \%) .\end{array}$ \\
\hline Takano et al. 24 & 2002 & $\begin{array}{l}\text { Shanghái } \\
\text { (China) }\end{array}$ & 1995-1998 & $\begin{array}{l}\text { Unidad } \\
\text { administrativa } \\
\text { (20) }\end{array}$ & 1 & $\begin{array}{l}\text { La razón de mortalidad estandarizada mostró una } \\
\text { relación estadísticamente significativa con el indicador } \\
\text { de privación y con otros indicadores como cuidados } \\
\text { sanitarios y entorno residencial urbano. }\end{array}$ \\
\hline $\begin{array}{l}\text { Ruiz-Ramos et } \\
\text { al. } 25\end{array}$ & 2004 & $\begin{array}{c}\text { Sevilla } \\
\text { (España) }\end{array}$ & 1994-1998 & $\begin{array}{c}\text { Zona básica de } \\
\text { salud (33) }\end{array}$ & 1 & $\begin{array}{l}\text { La mortalidad aumenta significativamente a mayor } \\
\qquad \text { porcentaje de desempleo: } \\
\text { Hombres: mortalidad total, cáncer de pulmón, SIDA, } \\
\text { bronquitis y enfisema, cirrosis y causas externas. } \\
\text { Mujeres: diabetes, SIDA, cardiopatía isquémica, } \\
\text { bronquitis y enfisema y cirrosis. }\end{array}$ \\
\hline $\begin{array}{l}\text { Domínguez- } \\
\text { Berjón et al. } 13\end{array}$ & 2005 & $\begin{array}{l}\text { Barcelona } \\
\text { (España) }\end{array}$ & 1987-1995 & $\begin{array}{c}\text { Distrito (10); } \\
\text { Sección censal } \\
\text { (1.812) }\end{array}$ & 2 & $\begin{array}{l}\text { Incremento en la mortalidad por peor nivel } \\
\text { socioeconómico. } \\
\text { Al considerar cada distrito por separado esta } \\
\text { asociación se observó en la mayoría de ellos. }\end{array}$ \\
\hline $\begin{array}{l}\text { Domínguez- } \\
\text { Berjón \& } \\
\text { Borrell } 14\end{array}$ & 2005 & $\begin{array}{c}\text { Barcelona } \\
\text { (España) }\end{array}$ & 1987-1995 & $\begin{array}{c}\text { Sección censal } \\
(1.814)\end{array}$ & 1,2 & $\begin{array}{l}\text { Las áreas con peores condiciones de desempleo, } \\
\text { educación, clase social y un índice compuesto por las } \\
\text { tres, presentan mayor riesgo de mortalidad, tanto en } \\
\text { hombres, como en mujeres. El efecto fue mayor en } \\
\text { hombres. }\end{array}$ \\
\hline Singh et al. 26 & 2005 & $\begin{array}{l}\text { Moradabad } \\
\text { (India) }\end{array}$ & 2001 & $\begin{array}{l}\text { Unidad } \\
\text { administrativa } \\
\text { (60); Calle (306) }\end{array}$ & 3 & $\begin{array}{l}\text { Las muertes por infecciones se asociaron a clases bajas } \\
\qquad \text { en ambos sexos. } \\
\text { Las clases sociales altas se asociaron estadísticamente } \\
\text { a mortalidad por enfermedades circulatorias } \\
\text { en ambos sexos. } \\
\text { Las muertes por cáncer no se asociaron con la clase } \\
\text { social, excepto el cáncer de mama que se asoció con } \\
\text { mujeres de clase social alta. }\end{array}$ \\
\hline $\begin{array}{l}\text { Lemstra } \\
\text { et al. } 15\end{array}$ & 2006 & $\begin{array}{l}\text { Saskatoon } \\
\text { (Canadá) }\end{array}$ & 2001 & $\begin{array}{c}\text { Sección censal } \\
\text { (6) }\end{array}$ & 2 & $\begin{array}{c}\text { Nivel socioeconómico bajo vs. resto = 1,04 }(0,86-1,26) \text {. } \\
\text { Nivel socioeconómico bajo vs. nivel socioeconómico } \\
\text { alto }=2,49(1,62-3,83) .\end{array}$ \\
\hline $\begin{array}{l}\text { Diez Roux et } \\
\text { al. } 27\end{array}$ & 2007 & $\begin{array}{c}\text { Buenos Aires } \\
\text { (Argentina) }\end{array}$ & 2003 & $\begin{array}{c}\text { Sección censal } \\
\text { (28) }\end{array}$ & 1 & $\begin{array}{l}\text { No poseer diploma estaba asociado positivamente } \\
\text { con la mortalidad por todas las causas, patologías } \\
\text { cardiovasculares y otras causas. Las patologías } \\
\text { respiratorias en mujeres y neoplasias en mujeres y } \\
\text { hombres no mostraron asociación. }\end{array}$ \\
\hline $\begin{array}{l}\text { De Godoy et } \\
\text { al. } 16\end{array}$ & 2007 & $\begin{array}{l}\text { São José do Rio } \\
\text { Preto } \\
\text { (Brasil) }\end{array}$ & 2002-2003 & $\begin{array}{c}\text { Sección censal } \\
(432)\end{array}$ & 2 & $\begin{array}{l}\text { Se observó un coeficiente de mortalidad cardiovascular } \\
\text { un } 40 \% \text { superior en los sectores con peor nivel } \\
\text { socioeconómico. } \\
\text { Las ratios de mortalidad fueron similares en los niveles } \\
\text { socioeconómicos medio-bajos, medio-altos y altos. }\end{array}$ \\
\hline
\end{tabular}

(continua) 
Tabla 1 (continuación)

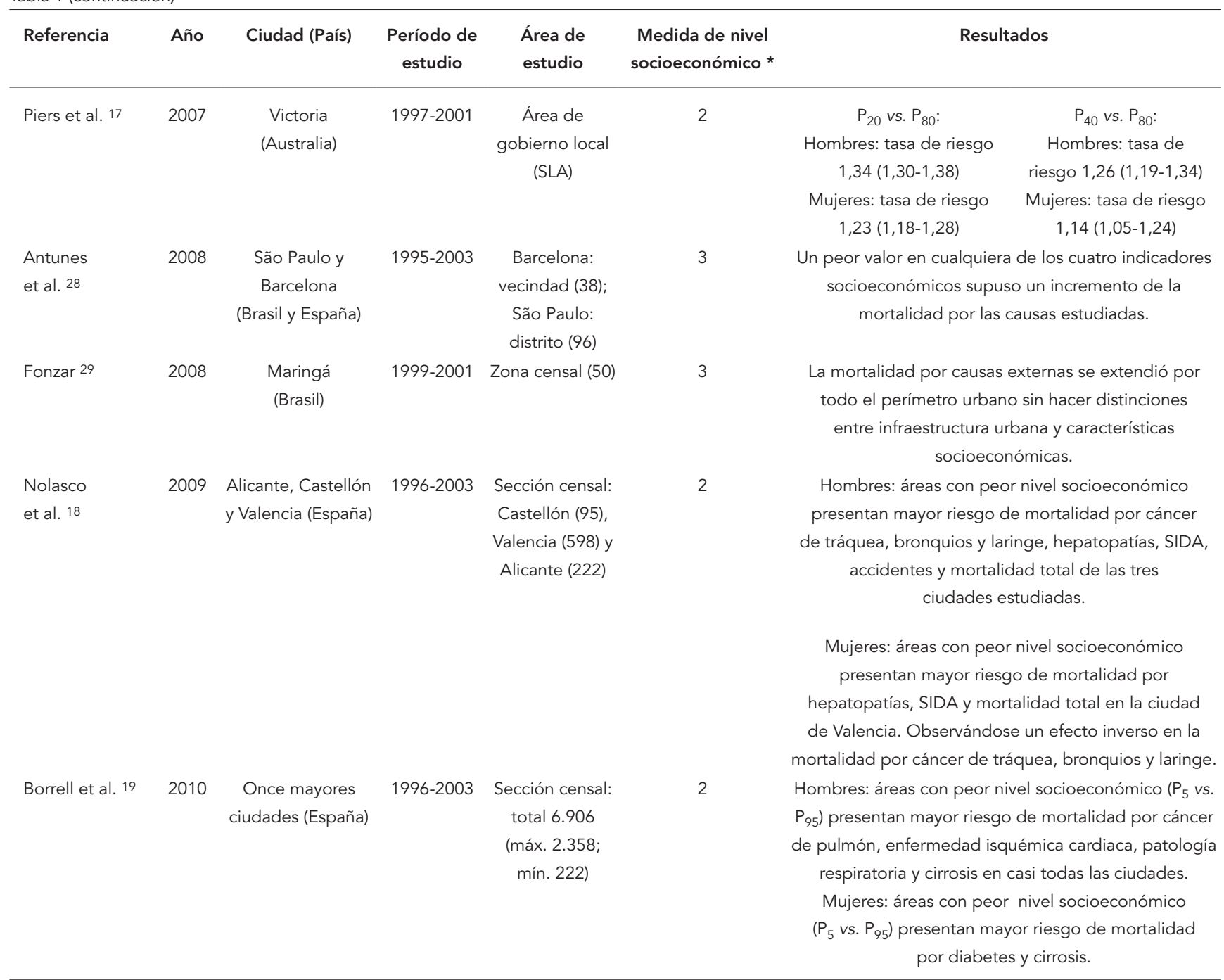

* Índice de privación: (1) indicador simple, (2) indicador compuesto, (3) varios indicadores simples, (4) asumen peores condiciones socioeconómicas en South Bronx.

\section{Resultados}

En la búsqueda bibliográfica, hasta junio de 2012, se obtuvieron 1.509 registros, 343 de ellos duplicados, de los que, tras una primera lectura de títulos y resúmenes (abstracts), se incluyeron 77 referencias para su lectura a texto completo. Tras la lectura completa de estos artículos se decidió incluir 24, ya que los 53 restantes no cumplían criterios de inclusión (Figura 1).

\section{Estudios incluidos}

Los estudios incluidos eran de diversa procedencia: once de ellos se realizaron en ciudades europeas, tres en ciudades australianas, cinco en ciudades de Sudamérica, dos de Norteamérica, dos asiáticas y uno presenta información de Barcelona (España) y São Paulo (Brasil) (Tabla 1).

Las ciudades estudiadas presentaban características muy diversas, en cuanto a población, áreas de estudio en las que se dividieron y periodo temporal del estudio (Tabla 1). Esta variabilidad permite extraer información con una gran validez externa.

\section{Metodología y variables de los estudios incluidos}

Los estudios incluidos, todos ellos con diseño de estudio ecológico, empleaban diferentes indicadores para valorar el nivel socioeconómico. Doce de ellos crearon un índice compuesto a partir de varios indicadores de privación simples 7,8,9,10,11, 
Tabla 2

Instrumentos utilizados para medir el nivel socioeconómico.

\begin{tabular}{|c|c|c|c|}
\hline Autor & Año & Ciudad (País) & Instrumento utilizado para medir el nivel socioeconómico \\
\hline Pringle et al. 20 & 1983 & Belfast (Irlanda) & Clase social (5 categorías). \\
\hline Byth et al. 7 & 1992 & Sídney (Australia) & Índice ABS (compuesto) y 5 indicadores simples. \\
\hline Martin Santos et al. 21 & 1992 & Málaga (España) & 26 indicadores simples (sociales y de salud). \\
\hline Siskind et al. 8 & 1992 & Brisbane (Australia) & Índice compuesto (educación, ingresos y ocupación). \\
\hline Arias et al. 22 & 1993 & Barcelona y Valencia (España) & $\begin{array}{l}\text { Indicadores simples: desempleo, analfabetismo y número de } \\
\qquad \text { coches } / 100 \text { habitantes. }\end{array}$ \\
\hline Bock \& Gans 9 & 1993 & Ciudad de Panamá (Panamá) & $\begin{array}{l}\text { Índice compuesto (análisis de componentes principales de } 47 \\
\text { indicadores socioeconómicos y demográficos). }\end{array}$ \\
\hline Fang et al. 30 & 1995 & New York (Estados Unidos) & $\begin{array}{l}\text { Se compara South Bronx con el resto de New York City. Se comenta } \\
\text { que los residentes son más jóvenes, pobres, con menor educación, } \\
\text { mayor desempleo y más minorías étnicas. }\end{array}$ \\
\hline Reijneveld 10 & 1995 & Ámsterdam (Holanda) & Índice compuesto (ingresos, desempleo y nivel educativo). \\
\hline Akerman et al. 11 & 1996 & São Paulo (Brasil) & $\begin{array}{c}\text { Índice compuesto (renta familiar per cápita, \% analfabetismo o con } \\
\text { primaria incompleta, \% viviendas con alcantarillado, personas por } \\
\text { domicilio y consumo de agua per cápita). }\end{array}$ \\
\hline Brimblecombe et al. 23 & 1999 & Oxford (Inglaterra) & $\begin{array}{l}\text { Índice de Townsend e indicadores simples: vivienda propia, precio de la } \\
\text { vivienda, desempleo masculino, desempleo femenino y hogares } \\
\text { con ayudas. }\end{array}$ \\
\hline Middelkoop et al. 12 & 2001 & The Hague (Holanda) & $\begin{array}{c}\text { Índice compuesto (media de ingresos del cabeza de familia y \% } \\
\text { desempleo entre los } 15 \text { y } 64 \text { años). }\end{array}$ \\
\hline Takano et al. 24 & 2002 & Shanghái (China) & Índice compuesto de privación y 18 indicadores simples. \\
\hline Ruiz-Ramos et al. 25 & 2004 & Sevilla (España) & Indicador simple: porcentaje de desempleo en varones. \\
\hline Domínguez-Berjón et al. 13 & 2005 & Barcelona (España) & $\begin{array}{c}\text { Índice compuesto (\% desempleo, \% instrucción insuficiente, \% } \\
\text { trabajadores manuales). }\end{array}$ \\
\hline Domínguez-Berjón \& Borrell 14 & 2005 & Barcelona (España) & $\begin{array}{c}\text { Índice compuesto (\% desempleo, \% instrucción insuficiente, \% } \\
\text { trabajadores manuales). }\end{array}$ \\
\hline Singh et al. 26 & 2005 & Moradabad (India) & $\begin{array}{l}\text { Índice compuesto (condiciones de la vivienda, educación, ocupación, } \\
\text { ingresos per cápita y bienes de consumo. }\end{array}$ \\
\hline Lemstra et al. 15 & 2006 & Saskatoon (Canadá) & Indicador simple: porcentaje de familias con bajos ingresos. \\
\hline Diez Roux et al. 27 & 2007 & Buenos Aires (Argentina) & Indicador simple: porcentaje de residentes sin estudios secundarios. \\
\hline De Godoy et al. 16 & 2007 & São José do Rio Preto (Brasil) & $\begin{array}{l}\text { Índice compuesto (años escolarizado cabeza de familia, años } \\
\text { escolarizada mujer cabeza de familia, ingresos cabeza de familia, } \\
\text { ingresos de mujer cabeza de familia, personas analfabetas, mujeres } \\
\text { analfabetas y viviendas con más de } 5 \text { personas). }\end{array}$ \\
\hline Piers et al. 17 & 2007 & Victoria (Australia) & $\begin{array}{l}\text { Índice compuesto (IRSD) (ingresos, educación, desempleo, trabajos de } \\
\text { baja competencia y otras variables que reflejan desigualdades). }\end{array}$ \\
\hline Antunes et al. 28 & 2008 & $\begin{array}{l}\text { São Paulo (Brasil) y Barcelona } \\
\text { (España) }\end{array}$ & $\begin{array}{c}\text { Tasa de desempleo, \% instrucción insuficiente, \% de estudios } \\
\text { secundarios y HDI (índice de desarrollo humano formado por ingresos, } \\
\text { educación y esperanza de vida). }\end{array}$ \\
\hline Fonzar 29 & 2008 & Maringá (Brasil) & $\begin{array}{l}\text { Indicadores simples: abastecimiento de agua, recogida de basura, } \\
\text { presencia de baño, red de alcantarillado, fosa séptica, condición del } \\
\text { domicilio, ingresos y grado de escolarización. }\end{array}$ \\
\hline Nolasco et al. 18 & 2009 & $\begin{array}{l}\text { Alicante, Castellón y Valencia } \\
\text { (España) }\end{array}$ & $\begin{array}{c}\text { Índice compuesto (desempleo, nivel educativo, nivel educativo en } \\
\text { jóvenes, trabajadores manuales y trabajadores temporales). }\end{array}$ \\
\hline Borrell et al. 19 & 2010 & $\begin{array}{l}\text { Once mayores ciudades } \\
\qquad \text { (España) }\end{array}$ & $\begin{array}{c}\text { Índice compuesto (desempleo, nivel educativo, nivel educativo en } \\
\text { jóvenes, trabajadores manuales y trabajadores temporales). }\end{array}$ \\
\hline
\end{tabular}


Diagrama de flujo de referencias.

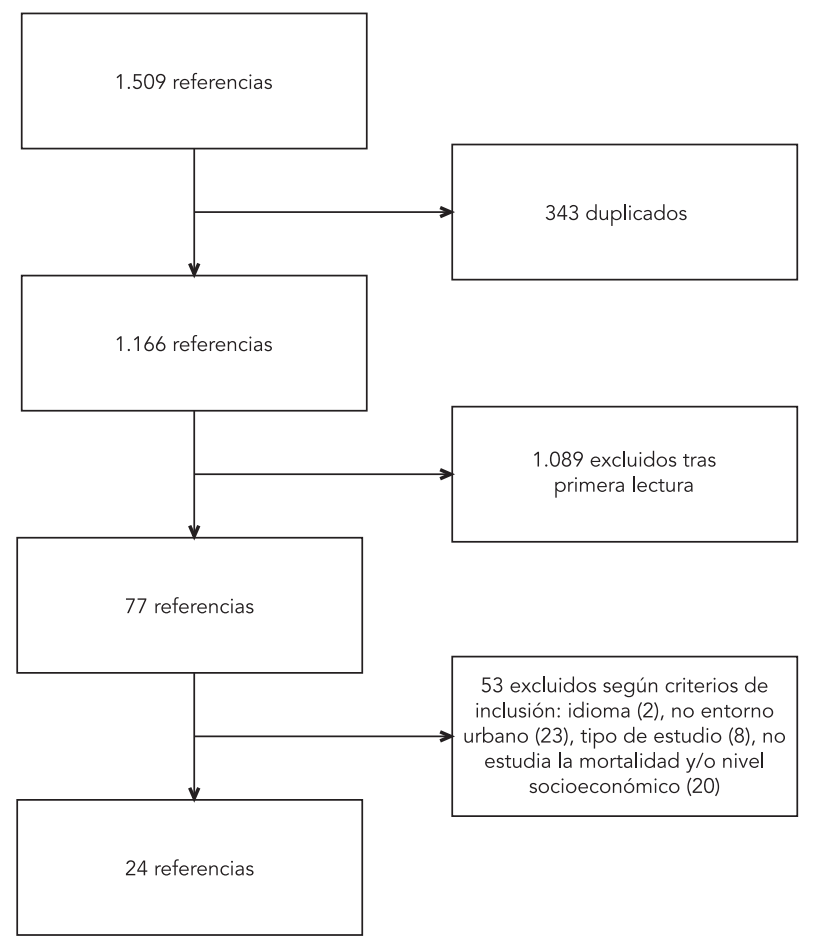

$12,13,14,15,16,17,18,19$, mientras que en once estudios $14,20,21,22,23,24,25,26,27,28,29$ la variable de privación era un indicador simple (Tabla 2). En todos ellos, el indicador de nivel socioeconómico, se utilizó para categorizar las áreas de las ciudades en diferentes niveles de privación.

Los artículos incluidos realizaron el análisis de la relación entre mortalidad y nivel socioeconómico con diferentes técnicas: correlación $7,20,22,23,24,28$, regresión lineal 8,10,12 o de Poisson $13,14,18,19,24,25$. Tres de los estudios 15,29,30 son descriptivos y únicamente valoran la distribución de la mortalidad, a partir de la distribución del nivel socioeconómico en las áreas de estudio (Tabla 1).

\section{Resultados de los estudios incluidos}

A pesar de las diferencias en el análisis y en la forma de recoger los datos de mortalidad y nivel socioeconómico, en general, todos los estudios mostraron una mortalidad más elevada en las áreas con peores indicadores de privación.

\section{- Patologías cardiovasculares}

De los 24 estudios incluidos, solamente ocho analizaban la asociación entre mortalidad y patologías cardiovasculares. En seis de ellos 11,16,19,20,27,30 se obtuvo que, en las áreas urbanas con peores indicadores socioeconómicos, existía mayor riesgo de mortalidad por cardiopatías. Sin embargo, en el trabajo publicado por Sing et al. 26, se observó una asociación inversa y en Ruiz-Ramos et al. 25 no se observaron diferencias significativas en dicho riesgo.

\section{- Neoplasias}

Los estudios incluidos categorizaban la mortalidad por cáncer de dos maneras: (a) mortalidad agregada por cáncer 20,26,27,30 y (b) mortalidad en función del tipo de cáncer 8,18,19,20,25,28.

Cuatro estudios 20,26,27,30 realizaron el análisis del riesgo de mortalidad por cáncer con la variable agregada. Solamente en el artículo de Fang et al. 30 se obtiene que la mortalidad es más elevada en las zonas de nivel socioeconómico más bajo 
para todas las franjas de edad (excepto en mayores de 75 años). En el resto 20,26,27 no se encuentra asociación entre mortalidad por cáncer y nivel socioeconómico.

Hay seis estudios que valoran la mortalidad por tipo de cáncer 8,18,19,20,25,28. El cáncer de pulmón presenta mayor mortalidad en clases sociales bajas 18,19,20,25, aunque Ruiz-Ramos et al. 25, Nolasco et al. 18 y Borrell et al. 19 la asocian también al sexo masculino.

En un estudio realizado en las ciudades de Barcelona y São Paulo ${ }^{28}$ se observó un incremento del riesgo de mortalidad en hombres por cáncer oral y faríngeo, a medida que aumentan la tasa de desempleo y el porcentaje de instrucción insuficiente, y que disminuye el nivel académico.

No se encontraron diferencias significativas en la mortalidad por cáncer colorrectal, de mama 8,25 o de próstata 19 entre los diferentes nivel socioeconómico.

\section{- Patologías pulmonares}

Cuatro 9,11,19,20 de los seis estudios que valoraron este grupo de patologías, encontrando que la mortalidad por patologías pulmonares aumenta de forma significativa a medida que disminuye el nivel socioeconómico en las ciudades. En los otros dos no se han encontrado diferencias 27,30.

Los estudios en los que se valoró únicamente la mortalidad por bronquitis 20 y neumonía 9,20 encontraron una elevada correlación con la clase social y una mayor incidencia y mortalidad prematura en las clases sociales bajas.

\section{- SIDA}

La relación entre mortalidad por SIDA y nivel socioeconómico se valoró en tres de los estudios incluidos 18,25,30. En los tres se obtuvo que la mortalidad por SIDA es significativamente mayor en zonas con nivel socioeconómico bajo.

\section{- Enfermedades infecciosas y parasitarias}

El riesgo de mortalidad por este tipo de enfermedades se analizó en tres estudios cuyas poblaciones son muy diferentes entre sí: Estados Unidos 30, la India 26 y Brasil 11. En todos ellos se aprecia una mayor tasa de mortalidad en las zonas con peores condiciones socioeconómicas. Todos los estudios consideraron como categoría de referencia los distritos con mayor nivel socioeconómico. En el artículo de Akerman et al. 11, además, se estimó el riesgo relativo de mortalidad en función del índice de privación. Los riesgos relativos (RR) que se obtuvieron son los siguientes: nivel socioeconómico 4 vs. nivel so- cioeconómico 3 ( $R R=1,41)$; nivel socioeconómico 4 vs. nivel socioeconómico $2(\mathrm{RR}=1,57) \mathrm{y}$ nivel socioeconómico 4 vs. nivel socioeconómico $1(\mathrm{RR}=2,10)$. No se presentaron los intervalos de confianza asociados a los RR.

\section{- Causas externas}

Tres trabajos, dos de ellos realizados en ciudades brasileñas 11,29 y otro en una española 25 , analizaron la mortalidad asociada a causas externas. En los estudios de Fonzar et al. ${ }^{29}$ y Ruiz-Ramos et al. ${ }^{25}$ se incluyeron como causas externas homicidios, accidentes, suicidios, caídas y ahogamientos, mientras que Akerman et al. 11 incluyó únicamente los homicidios y accidentes de tráfico. En el estudio realizado en Maringá (Brasil) 29 no se halló asociación, mientras que en el realizado en la ciudad de Sevilla (España) 25 se encontró una asociación significativa en hombres residentes en áreas con un porcentaje de desempleo mayor del 21\%. En el tercero, realizado en São Paulo 11, se comprobó que el riesgo de mortalidad por causas externas aumentaba a medida que disminuía el nivel socioeconómico del distrito de residencia.

\section{- Cirrosis}

Esta patología sólo se valoró en tres artículos, todos realizados en ciudades españolas 18,19,25. El que se llevó a cabo en Sevilla 25, obtuvo que la mortalidad por cirrosis en hombres era mayor en las zonas con un mayor porcentaje de varones desempleados. Tanto en hombres $(\mathrm{RR}=1,34$; IC95\%: 1,06-1,69), como en mujeres ( $R R=1,60$; IC95\%: 1,11-2,29), la mortalidad por cirrosis era mayor en las zonas urbanas con un porcentaje de desempleo en hombres mayor del $21 \%$.

En el estudio de Nolasco et al. ${ }^{18}$ se halló que en Valencia (España), existe mortalidad por cirrosis significativamente menor en las áreas urbanas de mayor nivel socioeconómico respecto a las de menor nivel, tanto en hombres, como en mujeres. Sin embargo, en Alicante (España), esta diferencia sólo es significativa en hombres.

En el estudio de Borrell et al. 19, en nueve de las once ciudades estudiadas se observó una asociación directa (en todas RR superiores a 2) entre la mortalidad por cirrosis en hombres y áreas con un bajo nivel socioeconómico. En las mujeres se observó esta asociación en siete de las once ciudades. 


\section{- Otras (abuso de drogas y alcohol, diabetes y muertes prevenibles)}

Fang et al. 30 evaluaron a existencia de incrementos en la mortalidad en función de los diferentes nivel socioeconómico por abuso de drogas y alcohol, y encontraron que la mortalidad por estas causas es mayor en las zonas más deprimidas para todos los grupos de edad y, tanto en hombres, como mujeres. Ruiz-Ramos et al. 25 , por su parte, valoraron la mortalidad por diabetes asociada al porcentaje de desempleo en hombres, y observaron que existen diferencias significativas en la mortalidad en las mujeres en aquellas áreas donde el desempleo supera el $21 \%$. En el estudio de Borrell et al. 19 también se observó asociación entre la mortalidad específica por diabetes y el nivel socioeconómico, especialmente en el caso de las mujeres, en ocho de las once ciudades estudiadas se encontró asociación, mientras que en los hombres en tan solo tres (las que presentaban mayor población).

Siskind et al. 8 analizaron la mortalidad por causas evitables en individuos con menos de 65 años de edad (se incluyeron en este grupo tuberculosis, cáncer de cérvix, enfermedad de Hodgkin, anemias, hipertensión, enfermedad aguda respiratoria, bronquitis, neumonía, asma, apendicitis, hernia y colecistitis) y observaron un incremento significativo en la mortalidad por estas causas a medida que aumentaba el nivel de privación.

Nolasco et al. 18 evaluaron la mortalidad por accidentes de tráfico en tres ciudades españolas y encontraron que existe más riesgo de mortalidad en hombres de zonas con peores indicadores socioeconómicos en las ciudades de Castellón y Valencia (RR = 3,6; IC95\%: 1,2-11,1 y RR = 1,8; IC95\%: 1,2-2,9, respectivamente). En Alicante, sin embargo, no se encontraron diferencias significativas en el resto de los estratos sociales, y tampoco en mujeres.

\section{Discusión}

Debido a la naturaleza de los estudios objeto de esta revisión, y con el fin de no omitir información relevante, la búsqueda bibliográfica se llevó a cabo de forma exhaustiva.

Los términos empleados en la estrategia de búsqueda, fueron "mortalidad" y "factores socioeconómicos" y por la baja especificidad de los mismos, se obtuvieron un gran número de artículos que trataban sobre temas no relacionados con el objetivo de esta revisión. No se utilizaron límites temporales para no perder información relevante, aunque ésta no fuera actual. A pesar de intentar abarcar toda la información disponi- ble, cabe la posibilidad de incurrir en el sesgo de publicación, debido a la tendencia de las revistas científicas -y de los propios investigadores- de darle menos importancia a los estudios con resultados no significativos que a los que obtienen resultados significativos.

Aunque los criterios de inclusión y exclusión no fueron excesivamente estrictos, permitieron descartar una gran parte de la bibliografía obtenida inicialmente, en parte debido a la baja especificidad de los términos de búsqueda. Otro factor derivado de los criterios de inclusión empleados es que todos los estudios incluidos en la revisión son de tipo ecológico, por ser el tipo de diseño que permite abordar la asociación entre nivel socioeconómico y mortalidad entre diferentes áreas en misma ciudad.

Como variable independiente se consideró el nivel socioeconómico. Hasta la fecha, no existe un consenso acerca de la forma ideal de valorarlo, por lo que en algunos estudios se emplea un indicador de nivel socioeconómico simple $7,8,9,11,12,13,15,16,17,18$ y otros $10,14,19,20,21,22,23,24,25,26$, 27,28,29, un índice complejo calculado a partir de varios indicadores simples. Durante los últimos años se han creado múltiples índices compuestos para valorar el nivel socioeconómico, como el Townsend et al. 31 , el Morris \& Carstairs 32 o el Carstairs 33, aunque no existe evidencia que justifique que sean los mejores en todas las condiciones. En muchas ocasiones un índice que discrimina perfectamente las diferentes zonas de una ciudad no tiene por qué funcionar igual en otra 23 . Además, existen artículos que demuestran que índices empleados en zonas urbanas no son de utilidad en zonas rurales 34 .

En general, la metodología empleada en estos estudios es muy heterogénea y en algunos de ellos de no muy buena calidad: las variables dependientes e independientes consideradas difieren mucho de un estudio a otro. El hecho de que se utilicen diferentes indicadores para valorar el nivel socioeconómico, y de que se lleven a cabo distintos métodos para considerar la mortalidad, hace que se complique tanto la interpretación de los datos, como la comparabilidad entre los diferentes estudios.

Doce estudios tuvieron en cuenta el sexo a la hora de analizar las muertes, mientras que otros doce valoraron la mortalidad agregada por sexos, lo que supone una limitación a la hora de evaluar los resultados e impide que se haga un análisis en profundidad de la mortalidad en función del sexo, información muy importante en este tipo de estudios, dada la diferente influencia que tiene el factor estudiado sobre el sexo 35 .

Los grupos de edad estudiados difirieron de unos estudios a otros, mientras que unos incluy- 
eron toda la población, en otros sólo se tuvo en cuenta la mortalidad hasta los 65 años y en otros no se analizó la mortalidad en menores de edad. Este hecho puede tener repercusión a la hora de estimar el efecto del nivel socioeconómico sobre la mortalidad por patologías crónicas o con una lenta progresión. Así, las zonas con un peor acceso a servicios sanitarios por distancias o condiciones sociales, presentarán una mortalidad prematura por estas patologías. Por esta razón, acotar el grupo de edad puede ser de interés para valorar los resultados de ciertas patologías. En el estudio de Fang et al. 30 se observa que la razón de muertes por $10^{5}$ habitantes para cánceres y cardiopatías es superior en el barrio de South Bronx para todos los grupos de edad excepto en mayores de 75 años, en el que se invierte esta tendencia tanto para hombres, como para mujeres.

Las ciudades incluidas presentan características muy diversas, tanto en términos poblacionales, como organizativos. Es obvio que no existen las mismas diferencias entre las clases sociales más desfavorecidas y las más favorecidas en ciudades de países donde estas desigualdades son muy acusadas y ciudades de países con menores desigualdades y, por tanto, también los efectos que estas diferencias pudieran tener sobre la mortalidad serán distintos.

Todos los estudios incluidos son de tipo ecológico por lo que presentan un nivel bajo de evidencia (nivel 2c) 36 y una serie de limitaciones, comunes a todos ellos, ya que no permiten hacer inferencias a nivel de individuos. Pese a que presentan una serie de ventajas: (1) existen factores contextuales del área geográfica que explican la salud en un contexto ambiental independientemente de los factores individuales, (2) la detección de áreas geográficas con peores indicadores socioeconómicos y de salud facilita la puesta en marcha de intervenciones. Además, la perspectiva brindada por este tipo de estudios se debe entender como una manera de abordaje de la epidemiología y la salud pública, cuyo objeto de interés son los grupos 37.

La relación entre el nivel socioeconómico y la mortalidad por patologías cardiovasculares fue significativamente superior en las zonas más deprimidas, en seis de los ocho estudios que la valoraron. Probablemente, la evolución de la enfermedad sea más rápida en las zonas con peores condiciones socioeconómicas, de ahí la mayor mortalidad prematura 20,30. En los niveles sociales más elevados, la mortalidad por patologías cardiovasculares ocurre a edades más avanzadas $11,16,20,30$ por factores relacionados con la facilidad del acceso a servicios sanitarios y a sus tratamientos.
En el estudio de Ruiz-Ramos et al. 25 no se observaron diferencias significativas en dicho riesgo, según los autores, esto se puede deber a las actividades preventivas y asistenciales que se llevan a cabo en las poblaciones que sufren con mayor intensidad este tipo de patologías. En el trabajo publicado por Sing et al. 26 , justifican la asociación inversa observada en que la población con mayor nivel socioeconómico también presentaba una mayor proporción de factores de riesgo, asociados a patología cardiovascular como la obesidad, hipertensión y consumo de tabaco.

Cuando se revisó la mortalidad por neoplasias, ninguno de los estudios que analizó todos los tipos de cáncer agregados encontró relación entre la mortalidad por esta causa y el nivel socioeconómico. Lo mismo ocurrió con el cáncer colorrectal y el de mama. Debido a que estas patologías son de lenta evolución, y que se tuvieron en cuenta todos los grupos de edad, sería normal que no existiesen grandes diferencias entre las diferentes zonas de la ciudad, aunque sí existen diferencias en la edad de mayor mortalidad y, por tanto, en los años de vida perdidos.

Por su parte, las diferencias observadas en la mortalidad por cánceres orales, faríngeos y de pulmón entre zonas de mayor y de menor nivel socioeconómico en hombres podrían asociarse a la mayor exposición a sustancias y partículas perjudiciales, tanto en el plano laboral como en el mayor porcentaje de fumadores que suele asociarse a clases sociales más deprimidas 38 .

La clara asociación encontrada entre la mortalidad por SIDA y las zonas urbanas con peores condiciones socioeconómicas se explica por la relación de esta enfermedad con la adicción a drogas administradas por vía parenteral, y a estilos de vida poco saludables, más comunes en zonas con desestructuración social ${ }^{39}$. Algo semejante ocurre para la mortalidad por infecciones y/o parasitosis 11,26,30, que son causas de muerte muy asociadas a zonas más deprimidas y con peores condiciones de salubridad y saneamiento.

Varios han sido los tipos de análisis estadístico empleado, algo esperado por la heterogeneidad de los datos incluidos en los estudios. Los dos métodos más utilizados (excluyendo la descripción simple de datos) fueron el análisis de correlación y la regresión de Poisson. Ambos métodos parecen adecuados para el análisis de este tipo de datos, aunque cabe destacar que la regresión de Poisson aporta más información y, generalmente, de mejor calidad que la que aporta un análisis de correlación, siempre y cuando los datos recogidos permitan realizar el análisis. A pesar de que en este tipo de estudios, de áreas 
pequeñas con diseño ecológico, lo más correcto parece ser el empleo de métodos de suavizado de las razones de mortalidad estandarizada 40 , análisis que no se hizo en ninguno de los estudios incluidos.

En general, los estudios incluidos presentan resultados poco consistentes y limitaciones metodológicas importantes, que impiden tanto la comparación entre los estudios, como la extracción de conclusiones relevantes, referentes a la relación entre mortalidad y nivel socioeconómico. Parece esencial que en futuros estudios que se diseñen se tenga en cuenta el indicador de privación utilizado, la forma de recoger la mortalidad, poniendo especial interés en el grupo de edad, el sexo y la causa, y el tipo de análisis a emplear.

\section{Resumo}

As desigualdades socioeconômicas são uma causa de mortalidade e morbidade superior à maioria dos fatores de risco, especialmente no ambiente urbano. Fo realizada uma revisão sistemática da evidência científica, na qual foram incluídos artigos em inglês, espanhol, português e italiano, e da qual foram excluídos estudos de baixa evidência, onde não constava análise da relação entre mortalidade e nível socioeconômico no ambiente urbano. A seleção de artigos foi efetuada por dois revisores independentes e a extração de dados foi feita através de tabelas de evidência. Foram obtidos 1.509 registros e incluidos 24. Em todos os trabalhos, foi observada maior mortalidade nas áreas com os piores indicadores de privação. Observou-se uma associação com patologias cardiovasculares em seis estudos, com patologias pulmonares, em quatro deles, e com a AIDS, infecções, parasitoses e cirrose em três. Os estudos incluídos apresentam resultados pouco consistentes e importantes limitações metodológicas, impedindo a comparação entre estudos e a inferência de conclusões relevantes.

Saúde Urbana; Mortalidade; Desigualdades em Saúde; Fatores Socioeconômicos

\section{Colaboradores}

A. Salgado-Barreira ha participado en la búsqueda, selección y lectura crítica de los artículos incluidos, así como en la redacción y revisión del documento final. A. Estany-Gestal ha participado en la selección de artículos, extracción de la información y en la redacción y revisión de la versión final del artículo. A. Figueiras ha contribuido en la selección de artículos, cuando existía desacuerdo entre A.S.-B. y A.E.-G., y en la supervisión, redacción y revisión de la versión final del artículo. 


\section{Referencias}

1. Alabart A, García S, Giner S. Clase, poder y ciudadanía: a modo de introducción. Madrid: Siglo XXI de España Editores; 1994.

2. Price $\mathrm{CH}$, Tsouros A. Our cities, our future: policies and actions plans for health and sustainable development. Copenhagen: WHO Healthy Cities Project Office; 1996.

3. Commission on Social Determinants of Health. Closing the gap in a generation: health equity through action on the social determinants of health. Final Report of the Commission on Social Determinants of Health. Geneva: World Health Organization; 2008.

4. Borrell C, Peiró R, Ramón N, Pasarín MI, Colomer C, Zafra E, et al. Desigualdades socioeconómicas y planes de salud en las comunidades autónomas del Estado español. Gac Sanit 2005; 19:277-86.

5. Borrell C, Pasarín MI. Desigualdad en salud y territorio urbano. Gac Sanit 2004; 18:1-4.

6. Benach J. Atlas de mortalidad en áreas pequeñas en España (1985-1987). Barcelona: Merck; 2001.

7. Byth K, McIntosh JH, Piper DW. A note on sex-specific mortality and social class in Sydney, 1979 to 1983. Aust J Public Health 1992; 16:321-3.

8. Siskind V, Najman JM, Veitch C. Socioeconomic status and mortality revisited: an extension of the Brisbane area analysis. Aust J Public Health 1992; 16:315-20.

9. Bock S, Gans P. The problem of fuzzy cause-specific death rates in mortality context analysis: the case of Panama City. Soc Sci Med 1993; 36:1367-71.

10. Reijneveld SA. Causes of death contributing to urban socioeconomic mortality differences in Amsterdam. Int J Epidemiol 1995; 24:740-9.

11. Akerman M, Campanario P, Maia PB. Environment and health: an analysis of intra-urban differentials in the city of Sao Paulo, Brazil. Rev Saúde Pública 1996; 30:372-82.

12. Middelkoop BJ, Struben HW, Burger I, VroomJongerden JM. Urban cause-specific socioeconomic mortality differences. Which causes of death contribute most? Int J Epidemiol 2001; 30:240-7.

13. Domínguez-Berjón MF, Borrell C, Lopez R, Pastor V. Mortality and socioeconomic deprivation in census tracts of an urban setting in Southern Europe. J Urban Health 2005; 82:225-36.

14. Domínguez-Berjón MF, Borrell C. Mortality and socioeconomic deprivation in the census tracts and districts of Barcelona. Gac Sanit 2005; 19: 363-9.

15. Lemstra M, Neudorf C, Opondo J. Health disparity by neighbourhood income. Can J Public Health 2006; 97:435-9.

16. de Godoy MF, de Lucena JM, Miquelin AR, Paiva FF, Oliveira DL, Augustin Jr. JL, et al. Cardiovascular mortality and its relation to socioeconomic levels among inhabitants of Sao Jose do Rio Preto, Sao Paulo state, Brazil. Arq Bras Cardiol 2007; 88:200-6.

17. Piers LS, Carson NJ, Brown K, Ansari Z. Avoidable mortality in Victoria between 1979 and 2001. Aust N Z J Public Health 2007; 31:5-12.
18. Nolasco A, Melchor I, Pina JA, Pereyra-Zamora P, Moncho J, Tamayo N, et al. Preventable avoidable mortality: evolution of socioeconomic inequalities in urban areas in Spain, 1996-2003. Health Place 2009; 15:732-41.

19. Borrell C, Marí-Dell'olmo M, Serral G, MartínezBeneito M, Gotsens M; MEDEA Members. Inequalities in mortality in small areas of eleven Spanish cities (the multicenter MEDEA project). Health Place 2010; 16:703-11.

20. Pringle DG. Mortality, cause of death and social class in the Belfast urban area, 1970. Ecol Dis 1983; 2:1-8.

21. Martin Santos FJ, March Cerda C. Social inequalities in health in the city of Malaga. Gac Sanit 1992; 6:198-206.

22. Arias A, Rebagliato M, Palumbo MA, Bellver R, Ashton J, Colomer C, et al. Health inequalities in Barcelona and Valencia. Med Clin 1993; 100:281-7.

23. Brimblecombe N, Dorling D, Shaw M. Where the poor die in a rich city: the case of Oxford. Health Place 1999; 5:287-300.

24. Takano T, Fu J, Nakamura K, Uji K, Fukuda Y, Watanabe M, et al. Age-adjusted mortality and its association to variations in urban conditions in Shanghai. Health Policy 2002; 61:239-53.

25. Ruiz-Ramos M, Sánchez J, Garrucho G, Viciana F. Desigualdades en mortalidad en la ciudad de Sevilla. Gac Sanit 2004; 18:16-23

26. Singh RB, Singh V, Kulshrestha SK, Singh S, Gupta P, Kumar R, et al. Social class and all-cause mortality in an urban population of North India. Acta Cardiol 2005; 60:611-7.

27. Diez Roux AV, Green Franklin T, Alazraqui M, Spinelli H. Intraurban variations in adult mortality in a large Latin American city. J Urban Health 2007; 84:319-33.

28. Antunes JL, Borrell C, Pérez G, Boing AF, WünschFilho V. Inequalities in mortality of men by oral and pharyngeal cancer in Barcelona, Spain and São Paulo, Brazil, 1995-2003. Int J Equity Health 2008; 7:1-9.

29. Fonzar UJV. Análise espacial da mortalidade por causas externas no município de Maringá, Estado do Paraná, 1999 a 2001. Acta Sci Health Sci 2008; 30:145-54.

30. Fang J, Bosworth W, Madhavan S, Cohen H, Alderman MH. Differential mortality in New York City (1988-1992). Part two: excess mortality in the south Bronx. Bull N Y Acad Med 1995; 72:483-99.

31. Townsend P, Phillimore P, Beattie A. Health and deprivation. Inequality and the North. London: Routledge; 1988.

32. Morris R, Carstairs V. Which deprivation? A comparasion of selected deprivation indexes. J Public Health Med 1991; 4:318-26.

33. Carstairs V. Deprivation indices: their interpretation and use in relation to health. J Epidemiol Community Health 1995; 49 Suppl 2:3-8. 
34. Gilthorpe MS, Wilson RC. Rural/urban differences in the association between deprivation and healthcare utilisation. Soc Sci Med 2003; 57: 2055-63.

35. Borrell C, Garcia-Calvente MM, Martí-Boscá JV. La salud pública desde la perspectiva de género y clase social. Gac Sanit 2004; 18 Suppl 1:2-6.

36. Oxford Centre for Evidence-Based Medicine. OCEBM levels of evidence system. http://www. cebm.net/index.aspx?o=5653 (accedido el 20/ Jun/2013).
37. Susser M. The logic in ecological: II. The logic of design. Am J Public Health 1994; 84:830-5.

38. Marmot M. Social determinants of health inequities. Lancet 2005; 365:1099-104.

39. Shelton JD, Cassell MM, Adetunji J. Is poverty or wealth at the root of HIV? Lancet 2005; 366:1057-8.

40. Best N, Richardson S, Thomson A. A comparison of Bayesian spatial models for disease mapping. Stat Methods Med Res 2005; 14:35-9.

Recibido el 28/Ago/2013

Versión final presentada el 04/Mar/2014

Aprobado el 01/Abr/2014 